\title{
Acquiring Creative Knowledge For Knowledge-Based Systems
}

\author{
Zhengxin Chen \\ Department of Computer Science \\ University of Nebraska at Omaha \\ Omaha, NE 68182-0500, UiS.A. \\ email: cs061@.cs.unomaha.edu
}

\begin{abstract}
In this paper, driven from the consideration of combining computational creativity into knowledge-based systems, we point out the need for studying a kind of creative knowledge concerning strategic heuristics. The issue of acquiring creative knowledge from folklore is discussed in some detail and is illustrated by examples. The impact of such knowledge on the architecture of knowledge-based expert systems is also discussed.
\end{abstract}

\section{Keywords}

Computational creativity, strategic heuristics, knowledge-based systems, knowledge acquisition, folklore.

\section{Introduction}

Knowledge-based systems (particularly expert systems) have become very popular in various applications. A well-known project which is still in progress is the CYC project (Lenat and Guha, 1990). In addition, machine learning techniques have been incorporated for automated knowledge acquisition of knowledge-based systems (Michalksi, 1983).

Knowledge is an important aspect of intelligence; creativity is another one. However, although sometimes expert systems may produce unpredicted results, the basic philosophy is reusing existing knowledge rather than creating new knowledge. Expert systems are not necessarily creative, and in general, they are not. In fact, expert systems are aimed to reuse experts' 
knowledge and to reuse reasoning process as well. Reuse also exists at even higher levels; for example, by developing expert system shells ${ }^{1}$.

As noted by Friedel (1992), expert systems can be viewed as a reactionary activity of creativities of the 19th century:

For most of the nineteenth century, technical novelty was largely seen as the product of human ingenuity and was closely associated with the "genius" of individuals and of the nation or race. In the twentieth century, by contrast, we have come to look for new technology from institutions and individuals who are characterized not by their creativity, imagination, and brilliance, but by their ability to marshal "expertise"...

However, creativity has been studied in AI, although in most cases it was not studied in the context of knowledge-based systems. One possible reason is due to the complex nature of creativity. The recent discussion (Stefik and Smoliar, 1995) around Margaret Boden's book The Creative Mind (Boden, 1990) reveals how little we know about creativity, and how much yet remains to be done.

This paper is primarily an attempt to address some issues related to creativity and expertise. It is not intended to be a complete survey of this subject. Rather, we will focus on one particular issue which has been ignored (or at least has not been emphasized), as will be briefly explained below. Therefore, the main purpose is not to provide a solution, but to present the question (although we will also provide a proposal at the end of the paper).

In this paper we argue that there is a need to study a kind of knowledge which is closely related to creative activities. It is different from domaindependent knowledge because it is not restricted to any specific application domain. It also differs from non-domain knowledge such as metaknowledge, because it is not knowledge about knowledge. In addition, it is not commonsense knowledge because the latter is the common knowledge possessed by every school child and methods used to make obvious inferences from this knowledge (Davis, 1990). The knowledge related to creativity is much more complicated than this. It is the wisdom and heuristics demonstrated in unconventional problem solving processes.

\footnotetext{
${ }^{1}$ This is not to say that the activity of reuse itself cannot be creative. But this is not an issue to be investigated here.
} 
In the sections that follow, first we briefly summarize some existing work on creativity within $\mathrm{AI}$ and point out the need for incorporating creativity into knowledge-based systems (Section 2). In Section 3, after a brief review on the nature of heuristics, we present a retrospective approach for knowledge acquisition of creative knowledge, including a brief summary of acquiring heuristic rules from technical invention and its relationship with detective stories. At the heart of this paper is Section 4 where we discuss folklore as another important source for acquiring creative knowledge. Some examples are used to illustrate the basic idea. In Section 5 some related issues are discussed, including how to use creative knowledge and problem which may be encountered in acquiring such knowledge. An architecture of enriched knowledge-based systems combining creativity, machine learning and expertise is proposed (Section 6). Concluding remarks are given in Section 7.

\section{Creativity in AI Literature}

In this section we briefly summarize some existing work related to AI. Just like human creativity, creative activities carried out by intelligent agents may take the direction of discovery or invention.

\subsection{Creativity in discovery systems}

According to Price (1978), invention and discovery are very different processes. Among other things, he noted that when one discovers, in any age or society, there is a feeling akin to strong constraint because there seems to be only the one world to discover. With invention one surely has alternatives. Although we often think of invention as the result of applying some new piece of scientific understanding, no simple, direct relationship exists between highly constrained scientific discovery on the one hand and the free will of socioeconomic choice in the invention and innovation of technology on the other.

For discovery, the task is to reveal or uncover some existing features or relationships; while for invention, the task is to generate new solutions (or possible generate new problems as well). In the field of AI, computational creativity has been studied along with both the directions of discovery and invention. The volume by Langley $e t$ al. (1987) and the volume by Piatetsky- 
Shapiro and Frawley (1990) exemplify discovery systems. For example, given an appropriate set of data, a clever computer program can "rediscover" Kepler's law.

\subsection{Creativity in generative systems}

We now take a look at the direction of invention. Creativity is generally defined as the production of something (e.g., a scientific theory, work of art, poem, novel, etc.) which is both novel and valuable according to consensual judgment (Rothenberg, 1979). This definition requires innovative work and inventive minds. Computational creativity is typically realized by building generative systems which are able to produce some productions which demonstrate intelligence. Boden's work contains many interesting examples of generative systems (Boden, 1994; 1995). In a summary provided by Row and Partridge (1993), five necessary characteristics of computational creativity have been identified for building generative systems: it is necessary that knowledge is organized in such a way that the number of possible associations (the creative potential) is maximized; it is necessary to tolerate ambiguity in representations; there is a need for multiple representations; the usefulness of new combinations should be assessable; and all new combinations need to be elaboratable to find out their consequences.

\subsection{Creativity in decision support systems}

In the interdisciplinary area of decision support systems (where Al plays an important role), Holsapple and Whinston (1987) predicted that knowledge-based systems in future knowledge-based organizations should be "computer co-workers" with the ability to "recognize needs, stimulate insights and offer advice." Analogy and metaphor can provide an important role in offering advice. Research work has been conducted to support analogical problem solving or achieve creativity support systems through computerized metaphor generation. For example, in Young $(1987,1988)$, three levels have been defined for supporting metaphoric thinking: the secretarial level, the framework-paradigm level, and the generative level. The three support levels are hierarchical and cumulative; thus the generative level includes the prior two levels. A database method was developed for purposes of automatically generating metaphors at the request of the user. In addition, analogy has been incorporated into model management systems 
using case based reasoning (Liang, 1993). Metaphor or analogy has been studied from other perspectives related to decision making (Vranes et al., 1992; Marchant et al., 1993). Another experimental enquiry in creativity support systems can be found in Chen $(1993,1996)$.

\section{A Retrospective Approach for Acquiring Strategic Heuristics}

\subsection{Remarks on the nature of heuristics}

The studies of creativity in AI as summarized in the previous section are more like stand-alone research activities. In order to examine creativity in the context of knowledge engineering, there is a need for studying heuristic knowledge related to creativity.

Heuristics have been extensively studied by AI researchers. About a quarter of a century ago, McCarthy and Hayes distinguished between the epistemological and the heuristic problems within AI (McCarthy and Hayes, 1969, McCarthy, 1977), and a recent comment on the relationship between these two parts can be found in Ginsberg (1995). As for the nature of heuristics, Lenat (based on his AM and EURISKO programs) claimed that "(h)euristics are compiled hindsight, and draw their power from the various kinds of regularity and continuity in the world; they arise through specialization, generalization, and - surprisingly often - analogy" (Lenat, 1982). Some other researchers noted that "( $t$ )he history of Artificial Intelligence shows us that heuristics are difficult to delineate in a clear-cut manner and that the convergence of ideas about their nature is very slow" (Groner, Groner and Bischof, 1983). A comprehensive discussion on heuristics in Al can be found in Pearl (1984).

Heuristics have also been studied in knowledge-based systems, particularly around knowledge acquisition. Clancey proposed the heuristic classification problem-solving model (Clancey, 1985) and Chandrasekaran proposed a theoretical framework for looking at knowledge-based problem solving in terms of generic tasks (Chandrasekaran, 1986). Abstractly, the generic tasks can be characterized by providing information about a task specification in the form of generic types of input and output, domain knowledge and a family of control regimes. Six generic tasks have been found; they are considered very useful as building blocks for the construction (and understanding) of knowledge-based systems. Furthermore, rolelimiting methods have been studied (McDermott, 1988). In a sense, role- 
limiting methods provide a concrete way to realize generic tasks in knowledge-based reasoning.

\subsection{Studying strategic heuristics of creative knowledge}

However, heuristic knowledge as summarized above may not necessarily imply creativity. In order to combine creativity with knowledge as used in knowledge-based systems, there is a need to study heuristics which are related to creative knowledge. Since creative knowledge involved in intelligent behavior is huge and diverse, to focus our study we shall examine a kind of creative heuristics which may provide us with useful hints to achieve our goal in an unconventional way. Such knowledge can extend our domain knowledge, and enhance our reasoning ability. To distinguish this kind of heuristic knowledge from other forms of creative knowledge, due to the lack of an appropriate terms, in this paper we will informally refer to it as strategic heuristics ${ }^{2}$. The only reason to introduce this term is that our discussion on this kind of creative knowledge may not be applicable to other forms of creative knowledge.

\subsection{A retrospective approach for technical invention}

Another problem in current creativity study in $\mathrm{AI}$ as exemplified in generative systems is that they generate products in a rather ad hoc manner. To remedy this problem, a retrospective approach which puts emphasis on the analysis of historical events and past experiences for creative knowledge thus becomes eminent. Weber (1992) clearly indicated that his method of studying inventive minds in technical invention is a retrospective analysis and it is retrospective in a deep sense. Within computer science, Dasgupta (1994) provided an excellent explanation on Maurice Wilkes' invention of microprogramming; the approach used is also retrospective.

An important remark which must be added here is that a retrospective approach does not necessarily imply the lack of predictive power (so that it can provide a kind of guide for future study). Here we emphasize the retro-

\footnotetext{
${ }^{2}$ The meaning of the word "strategic" used here corresponds to the meaning of the noun strategy which is "the art or skill of using stratagems as in politics or business", while a stratagem is "a scheme designed to obtain a goal" (both from The American Heritage Dictitonary, 1983).
} 
spective examination because it provides an approach to acquire strategic heuristic knowledge.

In the following we briefly summarize Weber and Perkins' work in technical invention to illustrate the retrospective approach.

\subsubsection{Technical invention}

According to Weber (1992), two fundamental questions which need to be answered in technical invention are whether we can describe invention in a general way, and whether we can extract principles (heuristics) of invention from particular cases that have some generality across inventions.

For example, consider the transformation of the size increase applied to a spoon. A possible answer is shovel, but it has also added a shoulder and foot action. A heuristic which can be learned here is the following:

\section{Scaling heuristic:}

Generate inventions and ideas by changing size scale.

Another set of heuristics is concerned with joins, an operation which combines several things together if they share some common part. For example, a claw hammer is the join of a striker head and a claw that share a common handle. In addition, from the fact that "what the striker part of the hammer will do, the claw will undo, and vice versa" (Weber and Perkins, 1989), this example illustrates the following:

\section{Inverse join heuristic:}

Combine only those tools or ideas that are inverses of one another.

These principles or heuristic rules apparently indicate various strategies one can take; that is why we call this kind of creative knowledge obtained from analysis of technical invention strategic heuristics. Technical invention thus serves as an extremely important source of creative knowledge as well as the study of creativity.

\subsubsection{Acquiring creative knowledge from detective stories}

Another interesting source of creative knowledge is detective stories. Cawelti (1976) studied common patterns (or the "genre") of detective stories. In fact, detective stories have a great potential to become an important source for studying creativity. Unlike the cases in conventional AI 
where the goals are usually well-defined, frequently the goal of a mystery has a dynamic feature. For example, a goal may be to determine who is the murderer. However, during the course of the investigation, a related murder case occurred. Consequently, the original goal has to be revised. Solving a mystery is a process of performing heuristic search in a cognitive space. Due to space limitation, a more detailed discussion on detective stories will not be provided here. Nevertheless, we want to cite the following extract from Weber and Perkins (1992):

\begin{abstract}
Sometimes invention has very much the character of looking for a needle in a haystack. The inventors scrutinize innumerable possibilities, looking for what they want. In contrast, sometimes invention wears the persona of "Holmesian deduction." From a variety of clues, inventors piece together what must be the way to go. And, of course, one finds mixed forms between the haystack and Holmes.
\end{abstract}

\title{
4. Acquiring Strategic Heuristics from Folk Tales
}

\subsection{Folklore as a resource for studying creativity}

In addition to technical invention, creative ideas can also be found in other kinds of human intelligent activities, including the wisdom perceived in folklore, proverbs, idioms, folk tales, legends, parables, etc., in various cultures. In the rest of this paper we will examine some examples from folk tales. Following the lead of Weber and Perkins, we will examine some examples of folk tales and discuss how to obtain strategic heuristics from them. For example, how can you weigh an elephant without using a large size scale? How can you use a boat, a regular size scale, and a rich supply of rocks to measure the weight of an elephant? Folk tales can reveal useful and interesting unconventional problem solving techniques and provide a vivid source of creative knowledge.

In particular, in the following we will use some examples taken from Chinese folk tales to illustrate why folklore is a resource for studying creativity ${ }^{3}$.

${ }^{3}$ This section should not be viewed as a study of Chinese folklore, and the contents of the folk tales may not be accurate. 


\subsection{Examples}

\subsubsection{How to weigh elephants without using large size scales}

An old tribute to Cao Chong (or Tsao Chong, a son of general Cao Cao, or Tsao Tsao) in the 3rd century is concerned with the following problem: You want to know the weight of an elephant, but you do not have scales large enough to do the job. The solution provided by Cao Chong can be sketched as the following informal algorithm:

\section{Elephant weighing algorithm:}

Input: An elephant, a boat (which is strong enough to hold the elephant), a river to hold the boat (along with the elephant), a large enough supply of rocks, and a scale which can weigh rocks.

Output: the weight of the elephant

Method:

Steps:

1. Put the elephant in the boat, make a notch at the waterline.

2. Remove the elephant from the boat.

3. Put rocks into the boat until they reach the notch made in Step 1.

4. Loop

Each time remove a portion of rocks from the boat and weigh them in the scales; add up the weights until all rocks are removed and weighed.

5. Retum the final weight of the rocks, which is the weight of the elephant.

The method used in this algorithm employed the typical idea of divide and conquer, but other heuristics are also used. The heuristics learned here can be described at various levels. At one extreme, we may use direct variabilization to replace the constant "elephant" by a variable "thing". This is a simple use of generalization in induction (Michalski, 1983). At a much higher level, generalization can be used in full scale for all constants, including the measurement itself (that is, measurement can be in any form, not restricted to weighing). From this discussion, we may have the following two heuristic rules:

Replacement heuristic (lower level abstraction): If a thing is too heavy to be weighed, you can put it in a boat, mark the waterline, replace the heavy thing by rocks, remove a portion of the rocks and weigh, and take the sum. 
Replacement heuristic (higher level abstraction): In case you want to measure a thing $A$ which is not of a manageable size, if there is another thing $B$ which is of the same measurement as $A$ (without actually measuring). but can be divided into manageable pieces or components, then you can replace $A$ by $B$, measure these pieces separately, and take the sum.

\subsubsection{How to measure the area of an irregular map by weighing}

In the 1950s, the government of a newly established county in rural China wanted to know exactly how large the county is (namely, the area of the county). Although an accurate map was available, the task of measuring its area seemed to be quite challenging due to the irregular shape of the county. However, the problem was successfully solved, thanks to the wisdom of a carpenter. The idea is extremely simple, and can be sketched as the following algorithm.

\section{Map measuring algorithm}

Input: A map of the county A to be measured, a map of another county B with known area $a_{B}$ (the map is of the same scale as the map to be measured), a wooden board, a saw, scales used to weigh the wooden board, some glue (removable).

Output: Area of country A $\left(a_{A}\right)$

Method:

Steps:

1. Use the glue to paste the map of $A$ on a board made from wood, and use a saw to cut the map along the county boundary.

2. Weigh the wooden board shaped as the county; let $w$ denote the weight.

3. Divide the weight by the specific gravity of wood to calculate the volume of the wood.

4. Divide the volume by the thickness of the wood board. This results in a measure $m_{A}$ related to the area of the county - but not the absolute value of the size of the county.

5. Repeat steps 1 to 4 for county B for which the exact area is known; call the result $m_{B}$. Since $m_{B}$ is proportional to $m_{B}$ as the actual size of the unknown county $\left(a_{A}\right)$ to the actual size of the known county $\left(a_{B}\right)$, from the known values of $m_{A}, m_{B}$ and $a_{B}$, we can easily calculate $a_{A}$.

What is the strategic heuristic learned from this story?

Measurement change heuristic:

In case direct measurement is difficult, change it

to another kind of measurement. 


\subsubsection{How to win a horse race with poor horses}

The following story is credited to Tian Ji (or Tien Chi) during the period of Warring States in ancient China. Suppose you are engaged in a horse race with your rival. According to the racing rules, each competitor should play three times using his own horses which fall into three categories: good, average and poor horses. The horses in each category vary in quality, but all horses in the first category are faster than those in the second category, and all horses in the second category are faster than those in the third category. Whoever wins at least twice out of the three competitions will be declared the winner. You also know that your rival is rich and he can afford good horses; in fact his horse in each category is better than the one you have in each category. The problem is: given such unfavorable conditions, can you still find a strategy to win?

Assuming you are a good racer, you can still win if you follow Tian Ji's advice. Notice that the regulations do not require you to use your good horse to compete with your rival's good horse, your average horse to compete with his average horse, and so on. According to Tian Ji's strategy, you should use your poor horse to compete with the rival's good horse. Of course you will lose this round of the competition. However, you can still win the other two rounds by playing your good horse against the average horse of your rival, and your average horse against his poor horse.

What is the strategic heuristic learned from this folk tale? Earlier we indicated that heuristics can be obtained at different levels. Now we can point out that heuristics can be learned from different perspectives. One perspective is to view the horses used by each racer in three rounds of competition as a list ordered by the round of competition: good horse, average horse, and poor horse. A good strategy is thus concerned with a good pairing of the two lists. For example, the winning strategy can be considered as a pairing of the following two lists:

my poor horse
my good horse
my average horse

In general, for each permutation, we can determine whether it is good or not by pairing the horse at each position in the list with the horse of your rival at the corresponding position. In sum, we may have the following heuristic: 


\section{Permutation heuristic:}

If the possible answer consists of ordered lists, try to generate all possible answers by generating permutations and perform comparison by pairing them.

This heuristic requires computation which is not efficient. But we can try some other heuristics. Another lesson which can be learned from this present folk tale is that, in contrast to the idea behind greedy algorithms (in which we always take the short-sighted local maximal value), we will strive for the global optimal, and even make a voluntary sacrifice if necessary. The following heuristic summarizes this lesson at a very abstract level.

\section{Sacrifice heuristic: \\ Make a voluntary sacrifice from a global viewpoint.}

\subsubsection{How to convert a test of patience to a competition of speed}

The last example, also concerned with horses, may not be from China, but it demonstrates the same wisdom. In ancient times, a rich old man was dying. Just before he passed away he gave his will to his two sons. The statement found in the will was quite strange: The sons should ride horses away from home; the one whose horse gets back home last will fully inherit the father's property. Therefore, to receive the inheritance would seem to be a test of patience. The brothers wandered around the countryside with their horses, until one day a wise man offered a good idea to the brothers: Since the will requires that the winner's horse should get back home last, why don't you exchange your horses? In order to make your own horse arrive last, you should make your rival's horse arrive first. Therefore, after exchanging the horses, both brothers speeded up, so that a test of patience became a competition of speed.

What is the strategic heuristic learned from this folk tale?

Just like the folk tale studied before, there may be many ways to learn from this folk tale. One heuristic which can be learned is very similar to the one learned from the previous example, namely, generating all possible permutations and performing comparisons by appropriate pairing. A more direct lesson which can be learned from this example, however, is the following heuristic. If we view the horse and the rider as the container and the contents it holds, then we may have the following rule: 


\section{Container exchange rule:}

To reverse the relationship between two containers and their contents, exchange the containers.

\section{Discussion}

As a continuation of the previous section, this section addresses some issues related to creative knowledge acquisition.

\subsection{Difficulties and problems in acquiring strategic heuristics}

Based on what we have discussed in this section, it is time to point out some difficulties and problems in acquiring strategic heuristics.

First of all, not every folk tale has strategic value; in fact, most may not. So, how to determine the heuristic value of a folk tale?

The next problem is, suppose a folk tale is determined to have some strategic value, how does one actually extract the folk tale, and make it computationalized?

Similar to the case of inductive learning, another problem is overgeneralization (over-simplification). That is, the heuristics extracted from the folk tale may be too abstract, and the vivid color of the original story is lost. In this case, the heuristics extracted may not be as useful as one might have expected. In addition, at the current stage, how to extract the heuristics from a given folk tale is largely an art. For the time being, these heuristics can be entered manually by the programmers. However, in the long run, this process should be automated. To avoid over-generalization and manual heuristic entry, an alternative is to store original folk tales in a separate knowledge base. But to make use of the stored tales, an on-line analysis and understanding of stored folk tales is required, which could pose a very challenging task in natural language processing.

The reader may have also noticed that the heuristics learned from folk tales may not be accurate enough. In order to store strategic heuristics so that they can be useful for inference, some kind of formalization may be needed.

There are still some other difficulties. Our selection of folklore as the source of creative knowledge seems to be somewhat arbitrary. The reader may think that there may be a large number of sources for strategic heuristic 
knowledge. This is a very interesting observation. It is true that there may be many other possible sources for the study of creativity (one of them could be humor, for example). However, this does not necessarily mean that the number of sources is endless, because although human intelligent behavior can be characterized into many categories (folklore is one of them), the number of such categories is finite. But how to identify these sources? And are all these sources useful to us at all? Can the knowledge acquired from these sources be computationalized or need it be computationalized? We may ask ourselves endless questions, simply because our understanding about creative knowledge is so little. However, various problems encountered in creative knowledge acquisition are not an excuse for not studying strategic heuristics of creative knowledge at all. On the contrary, they provide an excellent challenge to researchers and practitioners in artificial intelligence and cognitive science.

\subsection{The nature of strategic heuristics}

Another important issue which must be addressed here is the nature of strategic heuristics and its relationship with the heuristics discussed in the beginning of Section 3. As explained in this paper, knowledge related to creativity can be acquired by an analysis of inventions in history, folklore, as well as other sources. However, we should keep in mind that possessing this kind of knowledge does not guarantee creativity (since it consists of heuristic rules). Besides, we should also remember that creativity is not a pure cognitive or mental activity, it is mixed with other factors (such as social factors). In addition, since in most cases we are not pushed to invent new things or ideas, this kind of knowledge is of secondary importance (not as fundamental as domain knowledge which is important in solving various real-world applications in our daily life), but could be very useful. The time has come to incorporate this kind of knowledge into knowledge-based systems.

Notice that this kind of creative knowledge is knowledge at the object level rather than the level of controlling the use of knowledge. If we follow the traditional separation of control knowledge (used by the inference engine) and the domain knowledge, then this kind of knowledge should be placed not with control knowledge. However, a knowledge base usually stores domain knowledge, which is different from the creative knowledge discussed here. Such a kind of knowledge also differs from meta knowledge, 
which is related to control knowledge. Therefore, as will be seen in the next section, the incorporation of creative knowledge may have an impact on the architecture of expert systems.

\section{Toward Knowledge-Based Architecture Combining Creativity and Expertise}

\subsection{Computational aspects of creativity}

If knowledge is power, and if there is a need to code encyclopedias into computers to be stored as knowledge, then why not store strategic heuristics as well? Due to the unique nature of creative knowledge, it should be stored in a separate knowledge base (separate from domain knowledge, meta knowledge, commonsense knowledge, etc.). Since acquiring such knowledge may be time consuming, the creative knowledge base can be constructed in an incremental manner. Introducing such a component will have some impact on the architecture of knowledge-based systems.

The ultimate goal of acquiring strategic heuristics and storing such kinds of rules is to use them as an aid to enhance the functions of knowledge-based systems. They can be combined with domain knowledge in various application domains so that interesting and novel conclusions or suggestions can be made, or the quality of the answer produced by the system can be improved. In general, creative knowledge will be consulted only when it is needed. For example, in case domain knowledge is not available (thus no conclusion can be made), creative knowledge may be consulted. Creative knowledge can also be used to deal with conflict resolution (that is, several rules in a rulebased system competing to be fired). In some cases, creative knowledge may be combined with domain knowledge to improve the quality of the answer.

Experienced researchers in human creativity have warned us that none of the methods of technical invention, "nor any of the related heuristics, will mechanically or computationally produce inventions. Human judgment is very much required. The disadvantage of relying on such judgment is the vagueness that comes with it. The advantage, as discussed in Weber and Perkins (1989), is that quite general heuristics may suffice to generate ideas, heuristics that would be completely unpalatable to computers and artificial intelligence. At this stage in the study of invention, that is a reasonable trade-off" (Weber, 1992). 


\subsection{Progress status}

The above seemingly pessimistic comments do not necessarily mean that we can or should do nothing at all in trying to computationalize creative knowledge. As pointed out by Schank and Foster (1995), "Some would argue that creativity is too broad and ill-defined to possibly hope for the development of a mechanistic theory. AI's standard reply is and has been: well, then, let's bite off a small chunk of it, come up with a simple theory (maybe even a rather stupid one), test it out, see where it breaks down, and try again."

This is the attitude we have taken. In fact, acquiring strategic heuristics of creative knowledge (as discussed in this paper) is just one aspect of our larger research topic which is concerned with the overall relationship between creativity and knowledge acquisition. Another aspect is how to incorporate creativity into the general task of the knowledge acquisition itself (which is generally considered as the bottleneck of knowledge engineering). Existing knowledge acquisition approaches typically provide users with some existing tools for knowledge acquisition, leaving very little room for the users to do creative work. It would be interesting to bring creativity into knowledge acquisition. Since, compared to the task of acquiring creative knowledge, this task is better defined, we have started implementing a prototype system, a self-evolving knowledge-acquisition tool which is able to improve its behavior over time. The tool allows the user (a domain expert) not only to enter new knowledge, but also to specify what kind of knowledge should be acquired and what kind of questions should be asked. In this way, an expert in a knowledge domain can always recast the tool for his own need so that "everybody gets whatever he wanted".

As for the task of acquiring strategic knowledge (the theme of this present paper), due to its unconventional nature, we believe a careful discussion and analysis is needed before any implementation is carried out. This paper serves as part of this purpose: bring the question to the table, and take a closer look at a kind of heuristic knowledge. We hope that such a kind of investigation will help us to understand whether the heuristic knowledge can (or should) be computationalized (or at least partly computationalized), and if part can be computationalized, how to make use of it. 


\subsection{An extended expert system model}

With the hope that creative knowledge can be used to enhance the reasoning ability of knowledge-based systems, we present in the following a proposal on how creative knowledge could be incorporated into knowledgebased systems and its impact on the architecture of these systems. Here we will follow the discussion from van Harmelen (1991) who distinguished three types of knowledge-based systems which are mixed up with objectlevel inference and meta-level inference:

- Reflect-and-act systems: the meta-level interpreter is called very frequently, before or after every object-level step.

- Crisis-management systems: the meta-level is called only if a crisis or an impasse occurs in the object level computation, for example when too many or not enough steps are possible at the object-level.

- Subtask-management systems: the metal-level knowledge is used to partition the object-level task into a number of subtasks.

Creative knowledge can be handled by a crisis-management approach, namely, the creative knowledge is consulted only if there is a need to do so. Following notations used by van Harmelen, if the conventional inference engine is the object level interpreter, we can introduce another interpreter to take care of the creative knowledge. The relationship of these components is shown in Fig. 1.

\section{Conclusion}

In summary, this paper is motivated by the following considerations:

- There is a need to combine creativity with expertise;

- we still know very little about creativity;

- but we should not wait until the nature of creativity is fully understood.

In particular, we described a retrospective approach to acquire strategic heuristic knowledge from folk tales. Studying such kinds of knowledge will improve our understanding of the nature of heuristics. In addition, we may take advantage of creative knowledge in building future intelligent systems. By adding a creative knowledge base consisting of strategic heuristics and 


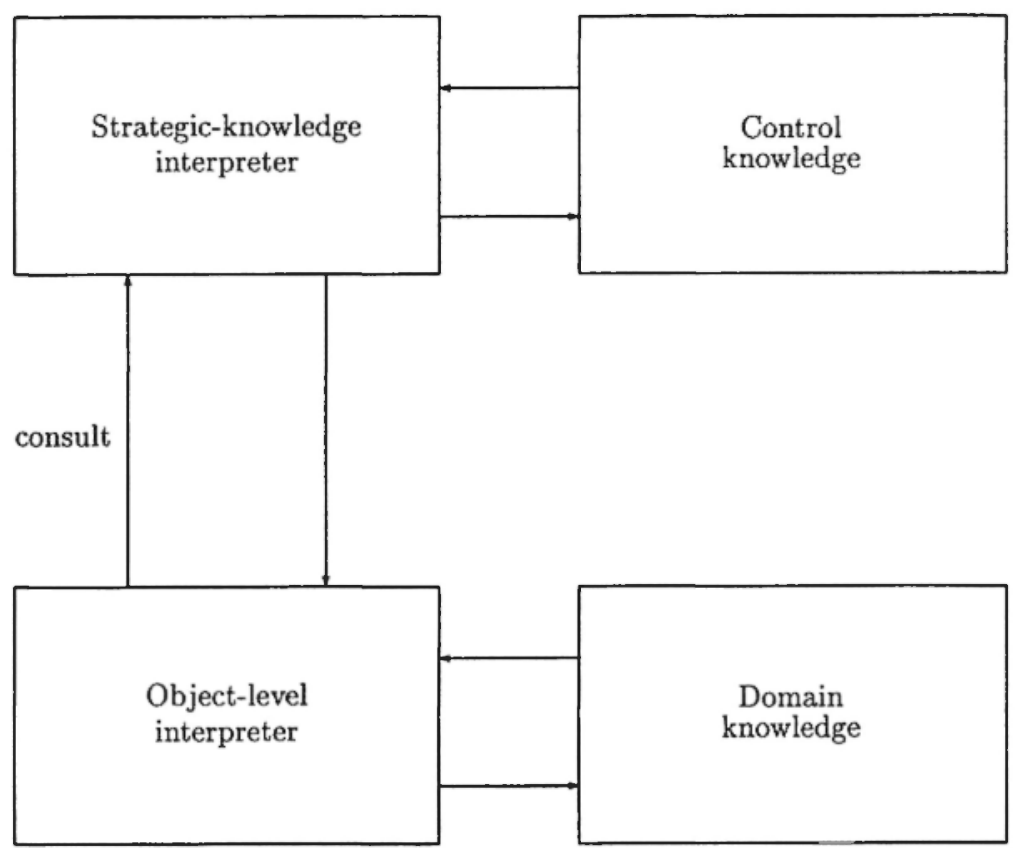

Fig. 1: Relationship between components.

its corresponding interpreter, a knowledge-based system should be able to enhance its reasoning ability. To achieve this end requires a lot of work. A comprehensive study between reasoning, judgment and creativity is needed.

\section{Acknowledgement}

The author thanks two anonymous reviewers whose critical comments on a previous version of this manuscript have helped the author to improve the paper.

\section{References}

Boden, M. Agents and creativity, Communications of the $A C M, 37$ (7), 117121, 1994.

Boden, M. Creativity and computers, Cybernetics and Systems, 26, 267-293, 1995. 
Boden, M. The Creative Mind: Myths and Mechanisms, London, Weidenfeld and Nicolson, 1990.

Cawelti, J.G. Adventure, Mystery, and Romance: Formula-Stories as Art and Popular Culture, Chicago, University of Chicago Press, 1976.

Chandrasekaran, B. Generic tasks in knowledge-based reasoning: High-level building blocks for expert system design, IEEE Expert, 1 (3), 23-29, 1986.

Chen, Z. Reasoning as extended retrieval, Communication and Cognition Artificial Intelligence (CC-AI), 10 (4), 343-356, 1993.

Chen, $Z$. Generating suggestions through document structure mapping, Decision Support Systems (1996, in press).

Clancey, W.J. Heuristic classification, Artificial Intelligence, 27, 289-350, 1985.

Dasgupta, S. Creativity in Invention and Design, New York, Cambridge University Press, 1994.

Davis, R. Representations of Commonsense Knowledge, San Mateo, CA, Morgan Kaufmann, 1990.

Friedel, R. Perspiration in perspective: changing perceptions of genius and expertise in American invention, in: Inventive Minds, Weber, R.J., Perkins, D.N. (eds.), New York, Oxford University Press, 1992; 11-31.

Ginsberg, M.L. Epistemological and heuristic adequacy revisited, $A C M$ Computing Surveys, 27 (3), 331-333, 1995.

Groner, R., Groner, M. and Bischof, W.F. (eds.). Methods of Heuristics, Hillsdale, NJ, Lawrence Erlbaum Associates, 1983.

Holsapple, C.W. and Whinston, A.B. Knowledge-based organizations, Information Society, 5, 77-90, 1987.

Langley, P., Simon, H.A., Bradshaw, G.L. and Zytkow, J.M. Scientific Discovery: Computational Explorations of Creative Process, Cambridge, MA, MIT Press, 1987.

Lenat, D.B. The nature of heuristics, Artificial Intelligence, 19, 189-249, 1982.

Lenat, D.B. and Guha, R.V. Building Large Knowledge-Based Systems: Representation and Inference in the CYC Project, Reading, MA, Addison-Wesley, 1990.

Liang, T.P. Modeling by analogy and case-based learning in model management systems, Decision Support Systems, 10, 137-160, 1993.

McCarthy, J. Epistemological problems of artificial intelligence, in: Proceedings of the Fifth International Joint Conference on Artificial Intelligence, Cambridge, MA, 1977; 1038-1044. 
McCarthy, J. and Hayes, P.J. Some philosophical problems from the standpoint of artificial intelligence, in: Machine Intelligence 4, Meltzer, B., Mitchie, D. (eds.), New York, Elsevier, 1969; 463-502.

McDermott, J. Preliminary steps toward a taxonomy of problem-solving methods, in: Automating Knowledge Acquisition for Knowledge Based systems, Marcus, S. (ed.), Boston, Kluwer, 1988; 120-146.

Michalski, R.S. A theory and methodology of inductive learning, Artificial Intelligence, 20 (2), 111-161, 1983.

Pearl, J. Heuristics: Intelligent Search Strategies for Computer ProblemSolving, Reading, MA, Addison-Wesley, 1984.

Piatetsky-Shapiro, G. And Frawley, W.J. Knowledge Discovery in Databases, Cambridge, MA, MIT/AAAI Press, 1991.

Price, D. De S. The evolution of invention, Part 6 in: The Smithsonian Book of Invention, Bourne, R. (ed.), New York, W.W. Norton \& Co., 1978; 184-190.

Rothenberg, A. The Emerging Goddess: The Creative Process in Art, Science, and Other Fields, Chicago, University of Chicago Press, 1979.

Rowe, J. and Partridge, D. Creativity: A survey of AI approaches, Artificial Intelligence Review, 7, 43-70, 1993.

Schank, R.C. and Foster, D.A. The engineering of creativity: a review of Boden's The Creative Mind, Artificial Intelligence, 79, 129-143, 1995.

Stefik, M. and Smoliar, S. (eds.). The Creative Mind: Myths and. Mechanisms: six reviews and a response, Artificial Intelligence, 79, 6567, 1995.

Van Harmelen, F. Meta-Level Inference Systems, San Mateo, Morgan Kaufmann, 1991.

Vranes, S., Lucin, M. and Stanojevic, M. Blackboard metaphor in practical decision making, Euro. J. Operations Research, 61 (1.2), 86-97, 1992.

Weber, R. Stone age knife to Swiss army knife: An invention prototype, in: Inventive Minds: Creativity in Technology, New York, Oxford University Press, 1992; 217-237.

Weber, R.J. and Perkins, D.N. How to invent artifacts and ideas, New Ideas in Psychology, 7, 49-72, 1989.

Weber, R.J. and Perkins, D.N. (eds.). Inventive Minds: Creativity in Technology, New York, Oxford University Press, 1992.

Young, L.F. The metaphor machine: A database method for creativity support, Decision Support Systems, 3, 309-317, 1987.

Young, L.F. Decision Support and.Idea Processing Systems, Dubuque, Wm. C. Brown Publisher. 